\title{
Exercise as Medicine for Mental and Substance Use Disorders: A Meta-review of the Benefits for Neuropsychiatric and Cognitive Outcomes
}

\author{
Garcia Ashdown-Franks ${ }^{1,2,3}$ (D) Joseph Firth ${ }^{4,5,6} \cdot$ Rebekah Carney $^{7} \cdot$ Andre F. Carvalho $^{8} \cdot$ Mats Hallgren $^{9}$. \\ Ai Koyanagi ${ }^{10}$. Simon Rosenbaum ${ }^{11,12}$. Felipe B. Schuch ${ }^{13}$. Lee Smith ${ }^{14} \cdot$ Marco Solmi $^{15}$. Davy Vancampfort ${ }^{16,17}$. \\ Brendon Stubbs ${ }^{1,2,3}$
}

๑) Springer Nature Switzerland AG 2019

\begin{abstract} ADHD, pre/post-natal depression). events were sparsely reported. symptoms across a broad range of mental disorders.

\section{Introduction}

In the general population, robust evidence indicates that physical activity (PA; any bodily movement that increases energy expenditure [1]) contributes to healthy ageing [2], improves sleep [3,4], and preserves cognition across the
\end{abstract}

Background Exercise may improve neuropsychiatric and cognitive symptoms in people with mental disorders, but the totality of the evidence is unclear. We conducted a meta-review of exercise in (1) serious mental illness (schizophrenia spectrum, bipolar disorder and major depression (MDD)); (2) anxiety and stress disorders; (3) alcohol and substance use disorders; (4) eating disorders (anorexia nervosa bulimia nervosa, binge eating disorders, and (5) other mental disorders (including

Methods Systematic searches of major databases from inception until 1/10/2018 were undertaken to identify meta-analyses of randomised controlled trials (RCTs) of exercise in people with clinically diagnosed mental disorders. In the absence of available meta-analyses for a mental disorder, we identified systematic reviews of exercise interventions in people with elevated mental health symptoms that included non-RCTs. Meta-analysis quality was assessed with the AMSTAR/+.

Results Overall, we identified 27 systematic reviews (including 16 meta-analyses representing $152 \mathrm{RCTs}$ ). Among those with MDD, we found consistent evidence (meta-analyses $=8$ ) that exercise reduced depression in children, adults and older adults. Evidence also indicates that exercise was more effective than control conditions in reducing anxiety symptoms (meta-analyses $=3$ ), and as an adjunctive treatment for reducing positive and negative symptoms of schizophrenia (metaanalyses $=2$ ). Regarding neurocognitive effects, exercise improved global cognition in schizophrenia (meta-analyses $=1$ ), children with ADHD (meta-analyses =1), but not in MDD (meta-analyses =1). Among those with elevated symptoms, positive mental health benefits were observed for exercise in people with pre/post-natal depression, anorexia nervosa/bulimia nervosa, binge eating disorder, post-traumatic stress disorder and alcohol use disorders/substance use disorders. Adverse

Conclusion Our panoramic meta-overview suggests that exercise can be an effective adjunctive treatment for improving

Electronic supplementary material The online version of this article (https://doi.org/10.1007/s40279-019-01187-6) contains supplementary material, which is available to authorized users.

Brendon Stubbs

brendon.stubbs@kcl.ac.uk

Extended author information available on the last page of the article lifespan [5-7]. Moreover, PA can positively influence neurogenesis in key areas of the brain [8-12]. For instance, longitudinal research [7, 13] and randomised controlled trials (RCTs) $[8,14]$ have demonstrated that PA can promote hippocampal neurogenesis in healthy and clinical populations. PA has also been positively associated with psychological wellbeing in children, adults and older adults [15-17]. Research has also demonstrated that PA can confer protection against the emergence of mental disorders. For example, higher levels of PA are consistently associated with a reduced risk of depression [18, 19], anxiety and stressrelated disorders [20, 21].

Exercise is a subset of the PA spectrum and is defined as a planned, structured, form of PA with the objective to 


\section{Key Points}

Across 27 reviews of various conditions, it was found that exercise reduces depression in children, adults and older adults, and is more effective than control conditions for reducing anxiety symptoms and for reducing positive and negative symptoms of schizophrenia.

There is also encouraging evidence for exercise in bipolar disorder, eating disorders and ADHD.

Results are promising and can be used as impetus to implement exercise programming into clinical practice.

improve or maintain physical fitness [1]. Whilst there is promising evidence that light intensity/free living PA has beneficial preventive effects in the general population (e.g. [22]), most treatment guidelines to date still focus solely on moderate-to-vigorous exercise (including resistance training) $[23,24]$.

Over the past 20 years, there has been a growing interest in the evidence and implementation of exercise as an adjunctive treatment among people with severe mental illnesses such as schizophrenia, bipolar disorder, major depressive disorder (MDD) and other mental illnesses such as pre/postnatal depression, anxiety and stress disorders, eating disorders (e.g. anorexia nervosa, bulimia nervosa, binge eating disorder, and alcohol and substance use disorders (alcohol use disorders and substance use disorders). In addition to the potential to help address the poor physical health and associated premature mortality in people with mental disorders [25-31], there is increasing recognition that exercise may have important effects on psychiatric symptoms [32-34] and neurocognitive functioning [32], across many conditions, thus potentially acting as a transdiagnostic treatment for mental disorders. This is important; whilst the pharmacological $[35,36]$ and psychotherapeutic approaches $[37,38]$ are helpful for many, they do not result in full remission in all patients. Furthermore, even in those who respond well to traditional treatments, some continue to experience residual symptoms, and/or a risk of future relapse [35, 36, 38]. Additionally, a broad spectrum of mental disorders are associated with cognitive dysfunction, for which current treatments are limited [39-43]. Thus, novel adjunctive treatments which improve mental health outcomes, whilst also targeting cognitive dysfunction, would provide a very promising approach for improving long-term outcomes across a range of mental disorders. Exercise may be well positioned to address this gap and act as a non-stigmatising intervention that can complement standard pharmacological and psychological interventions.
A plethora of systematic reviews and meta-analyses have synthesised the primary evidence for the potential for exercise to improve mental health symptoms across mental disorders. Consequently, international evidence-based recommendations for exercise in people with specific mental disorders have been developed and endorsed by key international organisations (e.g. [44, 45]). Despite this rapid expansion of meta-analytic evidence on exercise interventions for improving mental and cognitive outcomes in individual classes of mental disorders, no existing research has examined the potential efficacy of exercise as a transdiagnostic intervention across all classes of mental illness. Furthermore, there is little systematic examination of the evidence for aerobic, resistance and combined exercise modalities, which precludes translation into practice and policy. Moreover, the quality of these meta-analyses and the included trials has not been comprehensively evaluated, which is an indispensable step before more rigorous exercise recommendations are made. To address this gap within the literature, we set out to summarise and compare the existing top tier evidence from the most recent/largest, published meta-analyses of RCTs of exercise interventions targeting mental health and cognitive outcomes in people with mental illness. In the absence of a meta-analysis for a specific mental disorder, we identified systematic reviews of exercise in people with elevated mental health symptoms that included non-RCTs to provide a narrative synthesis.

\section{Methods}

\subsection{Searches}

Four independent pairs of authors searched MEDLINE/PubMed, PsycINFO and EMBASE from inception to 1/10/2018, for systematic reviews with meta-analyses of randomised controlled trials (RCTs) investigating exercise interventions (defined below) across a range of mental disorders.

Separate searches were undertaken for each mental disorder categorisation using the following standard terms: (exercise or aerobic exercise or PA or resistance training) and (systematic review or meta-analysis or meta*or metaanalytic review); and (1) (schizophrenia or psychosis or psychotic or major depression or depression or bipolar disorder or serious mental illness or serious mental disorder), or (2) (anxiety disorder or generalised anxiety disorder (GAD) or post-traumatic stress disorder (PTSD) or obsessive compulsive disorder (OCD) or panic disorder), or (3) (alcohol use disorder or alcohol addiction or substance use disorder or smoking or cigarette or drug addiction or addiction*) or (4) eating disorders (eating disorder or anorexia* or bulimia* or binge eating disorder) and others (ADHD or attention-deficit hyperactivity disorder or pre/post-natal depression). Reference lists of included articles were searched. 


\subsection{Inclusion Criteria}

We included meta-analyses of RCTs investigating any type of exercise in the following conditions: (1) serious mental illness (including schizophrenia spectrum, bipolar disorder and MDD); (2) anxiety and stress disorders (e.g. PTSD, OCD, GAD); (3) alcohol use disorders and substance use disorders, (4) eating disorders (anorexia nervosa, bulimia nervosa, binge eating disorder) and other mental disorders, including attention-deficit disorder (ADHD), pre/post-natal depression). Only meta-analyses including people with mental illnesses diagnosed through structured clinical diagnoses (e.g. DSM [46], ICD [47] criteria) were initially included. Meta-analyses that included people with mental illness/substance use disorders and mental health symptoms were only included if over $80 \%$ of the studies had a diagnosed mental illness. If this was not possible, we attempted to extract information on any subgroup meta-analysis results for those with confirmed mental illness. We included studies with data on young people, adults and older adults.

Exercise was defined activity that is planned, structured, and repetitive and has as a final or an intermediate objective the improvement or maintenance of physical fitness [1]. Within this definition, we included aerobic exercise (including brisk walking), high-intensity exercise, resistance training, mixed exercise (i.e. aerobic and resistance exercise). We did not include mind-body interventions such as yoga, tai chi and Pilates as the therapeutic benefit of these interventions is theoretically derived from components separate to the exercise itself $[48,49]$. We excluded low-intensity PA interventions (e.g. light walking, stretching) given the current focus of moderate-vigorous intensity exercise in current treatment guidelines [23, 24]. If we encountered meta-analyses with exercise and mind-body interventions or low-intensity PA, we included these if over $80 \%$ of the RCTs utilised exercise. We included meta-analyses that considered exercise studies used as monotherapy, or in combination with other types of treatment, e.g. psychotropic medication, psychological interventions.

Where no meta-analyses of exercise RCTs existed for a particular mental disorder, and thus no effect sizes could be extracted, we identified the most recent systematic review meeting the above criteria and summarised the main findings for exercise intervention (as defined above) in samples with clinical diagnoses of the disorder or those with elevated symptoms captured through validated tools.

\subsection{Primary Outcomes}

Primary outcomes were changes in psychiatric symptoms which characterise the target disorders, e.g. positive/negative symptoms in schizophrenia, depressive symptoms in MDD, anxiety levels in anxiety/stress disorders.

\subsection{Secondary Outcomes}

We also examined changes in secondary outcomes reported in meta-analyses of RCTs, such as comorbid psychiatric symptoms (e.g. depression in schizophrenia), neurocognitive function or associated changes in brain function or connectivity.

\subsection{Data Extraction}

Data extraction was undertaken by four pairs of authors. The information extracted comprised the number of studies included, the number of participants in each arm, participant demographics, length of follow-up, details of the exercise intervention, statistical analyses conducted, effect size information, heterogeneity (e.g. Cochran's $Q$ and the $I^{2} \%$ [50, 51]), publication bias (e.g. Egger's regression test [52]) and any meta-regression and subgroup analyses conducted. We did not undertake any additional analyses of the original statistical analyses.

\subsection{Risk of Bias (Quality) Assessment}

For meta-analyses of RCTs among people with structured diagnoses, two independent authors assessed the quality of the systematic reviews and meta-analyses using the AMSTAR and AMSTAR+ tool [53-55]. The AMSTAR is a reliable and valid tool to capture the methodological quality of meta-analyses but does not capture key quality indicators of the meta-analysed trials. Thus, in accordance with previous meta-reviews $[25,56]$ we used the AMSTAR+ which has six additional items on the content validity of included meta-analyses with scores ranging from 0 to 8 ( $>4$ indicating high quality).

\subsection{Strategy for Data Synthesis}

Due to the anticipated scale and heterogeneity of the literature, we summarised the results for each mental illness category using a best evidence synthesis and provided a summary of effect sizes reported across the meta-analyses. In the absence of meta-analyses of RCTs in those with structured diagnoses, we included systematic reviews with or without meta-analyses including people with mental disorders classified by validated symptom measures or other primary study designs.

\section{Results}

The initial searches identified 4089 de-duplicated hits, of which 269 full text screens were reviewed. Following the full-text screening, a total of 27 articles were included. This 
included 16 meta-analyses of 152 RCTs in people with clinical disorders [33, 34, 57-70] and 11 further systematic reviews in people with elevated symptoms and areas not covered by the existing meta-analyses [71-81]. The search results including reasons for exclusion are displayed in Fig. 1. A full list of excluded articles is available on request.

\subsection{Evidence from Meta-analyses of RCTs in Mental Disorders}

The key findings from each of the meta-analyses of RCTs among people with mental disorders categorised according to structured clinical diagnoses are summarised in Table 1.
The results with regard to each condition are presented below.

\subsubsection{Major Depression}

Eight meta-analyses examined effects of exercise in MDD [57-64]. The frequency, intensity, type and time of exercise varied considerably across the RCTs in the systematic reviews and invariably included a combination of aerobic with or without resistance training exercises. The mean AMSTAR and AMSTAR+ scores were 9.71 (range 7-11), 1.71 (range 1-4), respectively.
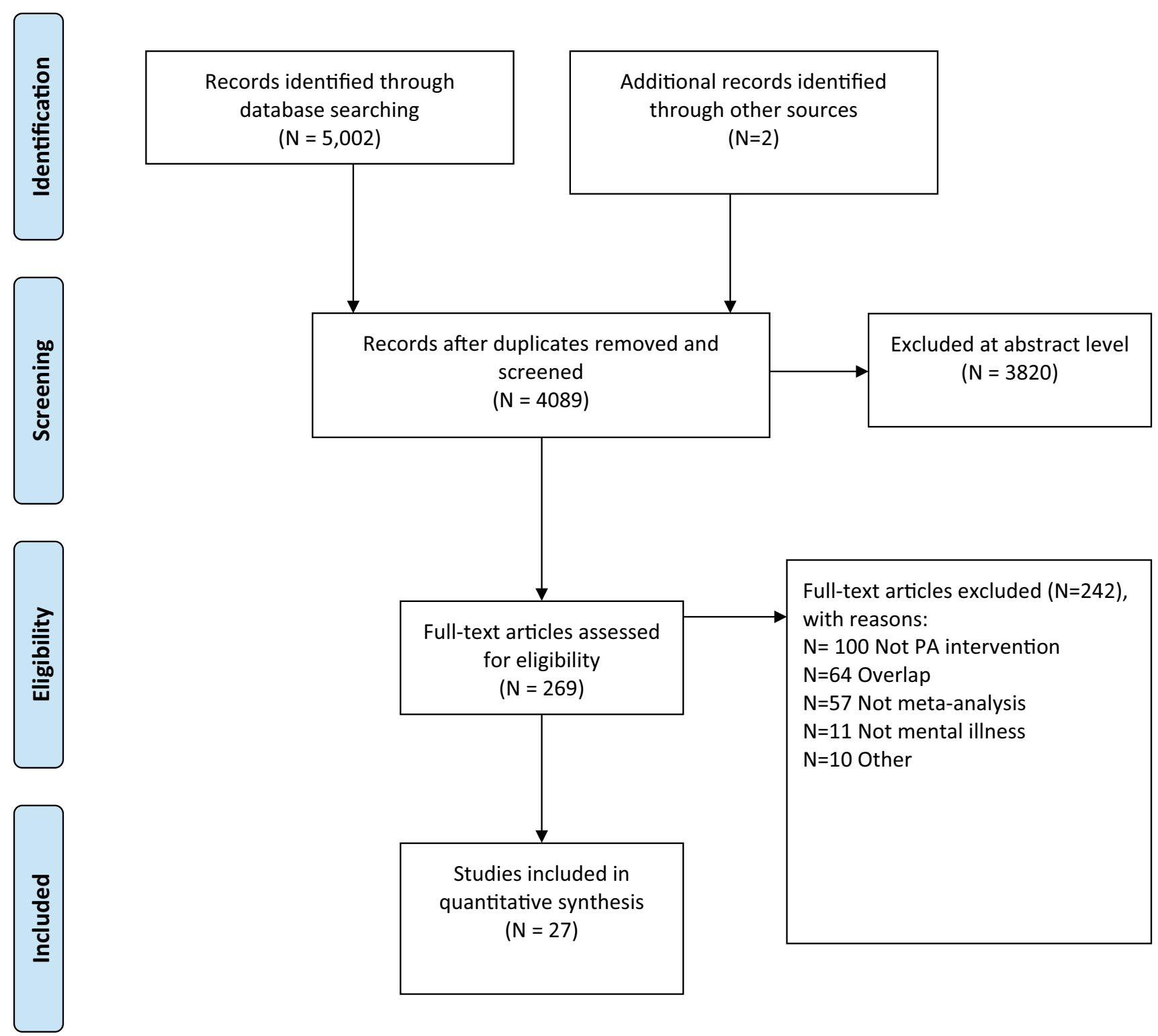

\section{Additional records identified through other sources} $(\mathrm{N}=2)$ $(N=5,002)$

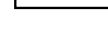

Fig. 1 PRISMA (2009) flow diagram for search strategy 


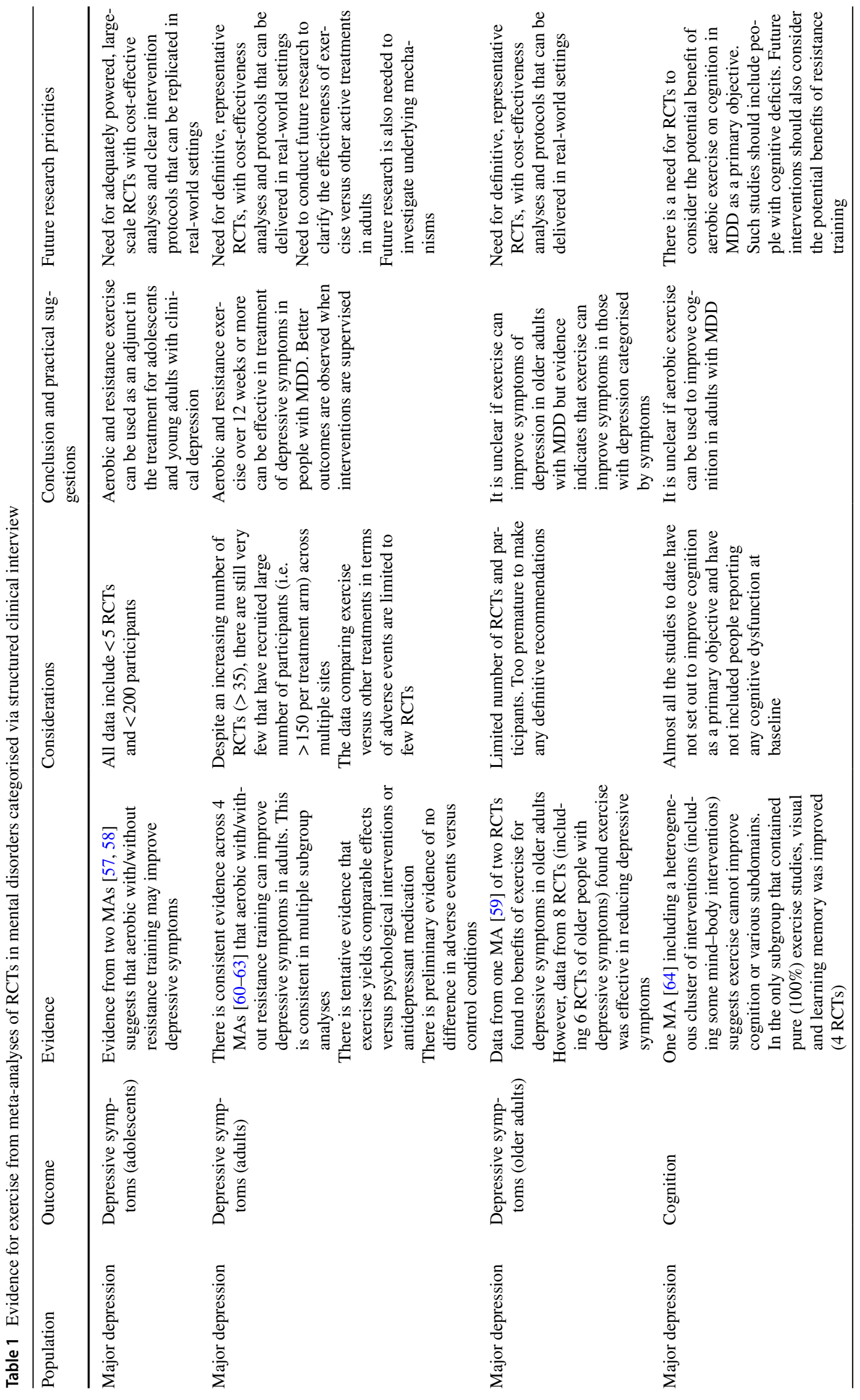




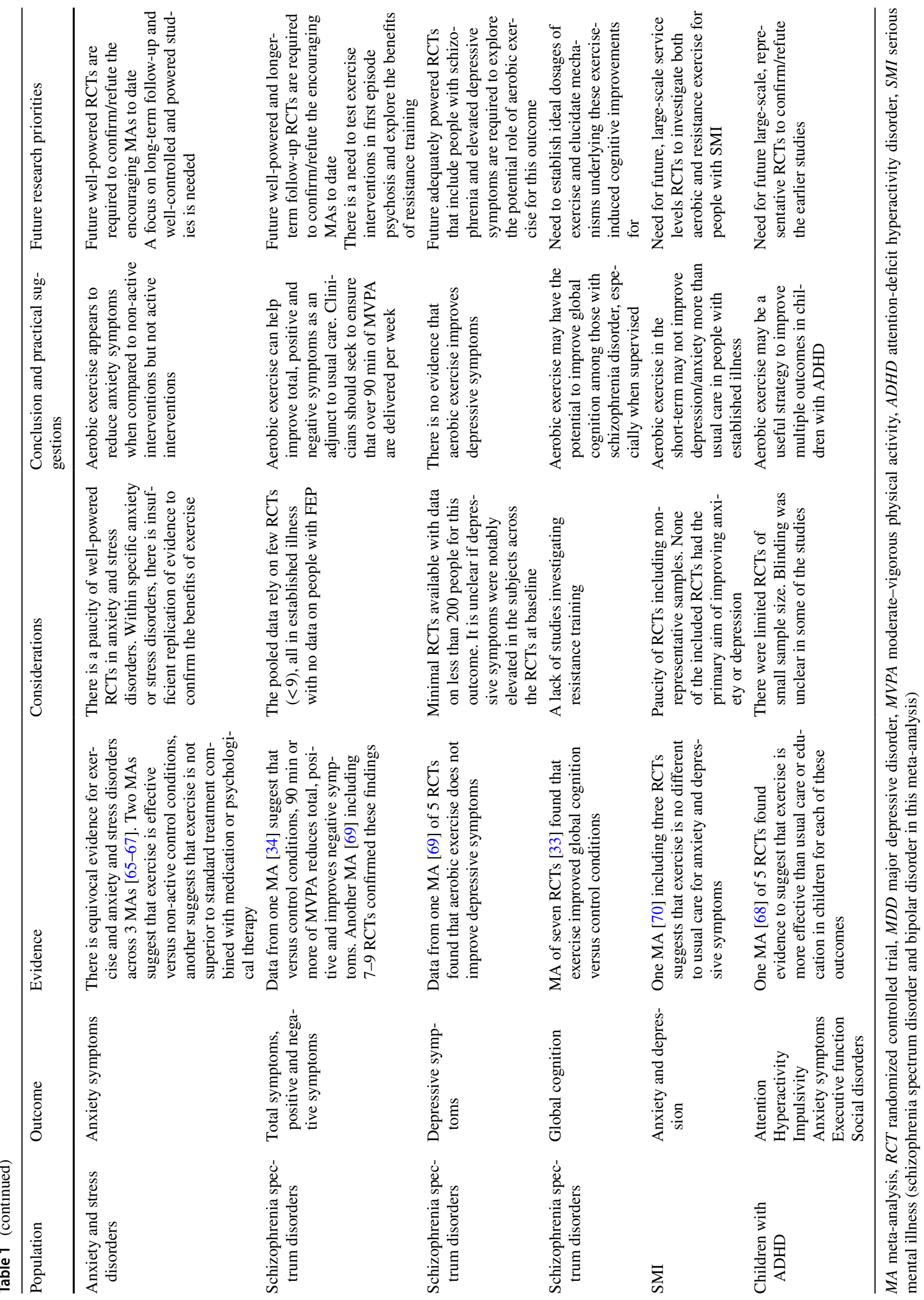




\subsubsection{Adolescents and Youth}

One meta-analysis [58] (AMSTAR $=7$ and AMSTAR $+=1$ ) reported the effects of exercise interventions across four RCTs of adolescent clinical samples with depression (mean age 16 years, $33 \%$ inpatients, $n$ intervention $=99, n$ control $=84)$. The results showed that aerobic and resistance exercise delivered over a median of 7 weeks (30-90 min, three times a week) had a moderately positive effect on symptoms of depression compared to control conditions including treatment as usual (TAU), stretching and instruction on health topics (SMD $=-0.43,95 \% \mathrm{CI}-0.84$ to $-0.02, p=0.04, I^{2}=44 \%$ ). Another meta-analysis [57] $($ AMSTAR $=10$, AMSTAR $+=2)$ which included adolescents and young adults with a clinical diagnosis of depression across four RCTs $(n=100)$ found exercise had a large effect on reducing depressive symptoms $(\mathrm{SMD}=-0.95$, $95 \% \mathrm{CI}-1.37$ to $\left.-0.53 p<0.00001, I^{2}=0 \%\right)$. Control conditions included no treatment, wait list and attention/activity placebo controls (i.e. stretching/flexibility, relaxation, physical education, very light activity and unguided group meetings).

\subsubsection{Adults}

Four meta-analyses of RCTs investigated the benefits of exercise for depressive symptoms in adults with MDD [60-63]. Information at a meta-analysis level regarding details of other treatments in the intervention groups (e.g. antidepressant medication, psychological therapy) was scant. The Cochrane review [62] (AMSTAR $=11$, AMSTAR $+=2$ ) included 23 RCTs of people with MDD and found that exercise reduced depressive symptoms $(\mathrm{SMD}=-0.57,95 \% \mathrm{CI}$ -0.81 to $-0.32, n=450, I^{2}=67 \%$ ). No further subgroup analyses were available for the MDD-only group.

Schuch et al. [60] $($ AMSTAR $=10$, AMSTAR $+=1)$ conducted a meta-analysis of aerobic or resistance exercise interventions for MDD and found that exercise had a large effect on improving depressive symptoms across 9 RCTs $\left(\mathrm{SMD}=1.139,95 \%\right.$ CI $0.464-1.814, p<0.0001, I^{2}$ $\%=88.54$ ) with a fail-safe number of studies to nullify the result of 123 . The results indicated large significant benefits when exercise interventions were supervised by qualified exercise professionals $(\mathrm{SMD}=1.537,95 \%$ CI $0.514-2.599$, $\left.p=0.003, I^{2} \%=91.61\right)$. However, the benefits of exercise were only evident in low-quality studies ( $\mathrm{SMD}=1.176,95 \%$ CI $\left.0.244-2.109, p=0.013 I^{2} \%=87.64\right)$. In that meta-analysis, 4 RCTs reported that $100 \%$ of intervention participants and controls were taking antidepressants, whilst 3 RCTs reported a portion of participations in both groups taking antidepressants.
Another meta-analysis [61] (AMSTAR $=10$, AMSTAR $+=1)$ included 23 RCTs $(n=977)$ with clinical depression ( 2 with treatment resistant depression and 4 with dysthymia); most included aerobic $(N=16)$ or resistance exercise $(N=4)$. Overall, exercise was effective in reducing depressive symptoms $(g=-0.68,95 \% \mathrm{CI}-0.92$ to -0.44 , $p=0.001, I^{2}=68 \%$ ). Effects were small, but still significant, after adjusting for publication bias $(g=-0.38,95 \%$ CI -0.62 to -0.13 ; fail-safe number 463 ). The benefits of exercise were evident in trials that reported blinding of outcomes $(g=-0.4,95 \%$ CI -0.7 to -0.11, RCTs $=10, n=600)$ and an ITT analysis $(g=-0.67,95 \%$ CI -0.9 to -0.44 , RCTs $=12, n=643$ ). However, across six RCTs with 461 participants that had blinded group allocation, blinded outcome assessments, and ITT analysis, a small, heterogeneous but non-significant effect was evident $(g=-0.26,95 \%$ CI -0.61 to $\left.0.08, p=0.14, I^{2}=68 \%\right)$. Aerobic exercise resulted in similar outcomes versus other active treatments including psychological treatment $(g=-0.22,95 \% \mathrm{CI}-0.65$ to 0.21 , RCTs $\left.=3, n=79, I^{2}=0 \%\right)$ and antidepressants $(g=-0.08$, $95 \% \mathrm{CI}-0.33$ to 0.18 , RCTs $\left.=3, n=236, I^{2}=0 \%\right)$.

In a trial sequential meta-analysis [63] (AMSTAR $=10$, AMSTAR $+=1$ ), exercise was more effective than control conditions across 35 RCTs (SMD $=-0.66,95 \% \mathrm{CI}-0.86$ to $-0.46, I^{2}=81 \%$ ). The benefits of exercise were evident in 31 RCTs deemed "high risk" of bias (SMD $=-0.75,95 \%$ CI -0.98 to $-0.52, n=1968, I^{2}=81 \%$ ). However, no difference was noted when comparing exercise versus other active interventions (including other forms of exercise) across four RCTs rated "high quality" (SMD $=-0.11,95 \% \mathrm{CI}-0.41$ to $0.18, n=530, I^{2}=62 \%$ ). Three RCTs reported adverse events and no difference in the exercise or control interventions. Four studies reported co-treatments; two used exercise in combination with sertraline, one used ECT and exercise and one used CBT and PA promotion along with exercise. Control conditions included meditation, occupational therapy, wait list, TAU, health education, sertraline, flexibility, placebo medication, stretching/relaxation, attention control, sertraline, behavioural activation.

Brondino et al. [64] (AMSTAR $=10$, AMSTAR $+=4)$ investigated the potential benefits of exercise for cognitive symptoms in MDD. Across several subgroup analyses exercise did not improve speed of processing; attention/ vigilance; working memory; verbal learning and memory and reasoning and problem solving. However, in a subgroup analysis that excluded mind-body interventions and included only exercise RCTs (four trials), the exercise improved visual learning and memory $(g=0.241,95 \%$ CI $0.005-0.478, I^{2}=68 \%$,). Control conditions included wait list, CBT, sertraline, placebo, relaxation training, stretching, and health education. 


\subsubsection{Older Adults}

One meta-analysis [59] (AMSTAR $=10$, AMSTAR $+=1$ ) included two RCTs of older people with MDD or dysthymia and found the effects of exercise on symptoms of depression fell short of statistical significance $(\mathrm{SMD}=-1.883,95 \% \mathrm{CI}$ 0.44 to $\left.-4.21, p=0.11, I^{2}=93.06 \%\right)$. However, the pooled data including older people with depressive symptoms found that aerobic exercise improved depressive symptoms $(\mathrm{SMD}=-0.90,95 \% \mathrm{CI}-0.29$ to $-1.51, \mathrm{RCTs}=8, n$ intervention $=138, n$ control $=129)$.

\subsubsection{Anxiety and Stress-Related Disorders}

Overall, three meta-analyses investigated the benefits of exercise in anxiety and stress-related disorders [65-67]. All systematic reviews were in adults and information at a metaanalysis level on additional treatments among participants (e.g. medication) was sparse. The frequency, intensity, type and time of exercise varied considerably across the RCTs. Overall, the mean AMSTAR and AMSTAR+ scores were 6.66 (range 5-9) and 3 (range 1-3), respectively.

Stubbs et al. [65] (AMSTAR $=6$, AMSTAR $+=1$ ) conducted a meta-analysis of exercise in six RCTs $(n=262$, mean 34.74 years) including panic disorder $(N=2)$, GAD $(N=1)$, PTSD $(N=2)$ and a further RCT that included people with either GAD, PD or social phobia. All but one RCT used aerobic exercise compared to non-active control conditions. Exercise reduced anxiety symptoms at the trial end point (SMD $=-0.581,95 \% \mathrm{CI}-1.0$ to $-0.76, p=0.02$, $I^{2}=66 \%$ ). Of the included RCTs, only 1 used exercise as a monotherapy, whilst another study reported $20 \%$ of participants and $20 \%$ of controls taking antidepressants. Information on other treatments in the other studies was not detailed.

An earlier meta-analysis [66] (AMSTAR $=5$, AMSTAR $+=2$ ) investigated the potential benefit of exercise versus non-active and active (antidepressant medication, non-aerobic exercise) in seven RCTs of 407 people with anxiety disorders. There was no benefit of aerobic exercise when compared to both treatment as usual or other active treatments for anxiety symptoms (SMD $=0.12,95 \%$ $\mathrm{CI}-0.33$ to $0.58, p=0.60$ ), although compared to wait list or placebo aerobic exercise was effective $(\mathrm{SMD}=1.42,95 \%$ CI $0.80-2.04, p=0.001$, RCTs $=2$ ). No difference was noted when stratified according to anxiety disorder type, when exercise was compared to pharmacotherapy ( $\mathrm{SMD}=-0.28$, $95 \% \mathrm{CI}-0.76$ to $0.20, p=0.25, \mathrm{RCTs}=2$ ) or versus $\mathrm{CBT}$ $(\mathrm{ES}=-0.70,95 \%$ CI $0.03-1.38, \mathrm{RCT}=1)$.

Aylett et al. [67] (2018, AMSTAR =9, AMSTAR $+=3$ ) meta-analysed four RCTs of people with anxiety disorders in primary care and found that exercise reduced anxiety symptoms ( $\mathrm{SMD}=-0.32,95 \% \mathrm{CI}-0.62$ to -0.01 ). Two studies used psychological therapy as co-treatments in both the intervention and control groups. Control groups included a non-active waiting list and a non-active control group taking placebos.

\subsubsection{Schizophrenia Spectrum/Serious Mental Illness}

Three meta-analyses investigated the benefits of exercise in schizophrenia spectrum disorders [33, 34, 69], whilst one investigated the benefits of exercise in people with serious mental illness [70]. All the systematic reviews were performed in adults. The frequency, intensity, type and time of exercise varied considerably across the RCTs in the metaanalyses. The AMSTAR and AMSTAR+ scores were all rated as 5 and 3 , respectively.

One meta-analysis [34] (AMSTAR $=5$, AMSTAR $+=3$ ) included 11 RCTs of exercise in schizophrenia spectrum disorders (mean 33 years (range $=25-52$ years, median illness duration 10 years)). Exercise interventions varied but only interventions delivered at over 90 min of MVPA per week improved total symptoms $(\mathrm{SMD}=-0.72$, $95 \% \mathrm{CI}-1.14$ to -0.29 ), positive symptoms (SMD $-0.54,95 \% \mathrm{CI}-0.95$ to -0.13 ) and negative symptoms $(\mathrm{SMD}=-0.44,95 \% \mathrm{CI}-0.78$ to -0.09$)$. In that meta-analysis, control conditions included TAU, behavioural therapy, table football, computer games, occupational therapy and wait list. Similar findings were evident for these outcomes in another meta-analysis [80] (AMSTAR $=5$, AMSTAR $+=3$ ), but no effect was found for depressive symptoms from aerobic exercise across five RCTs. A meta-analysis of seven RCTs [33] (AMSTAR = 5, AMSTAR $+=3$ ) of aerobic exercise and 292 people with schizophrenia spectrum disorder found that exercise improved global cognition versus control conditions which included table football alone $(N=1)$, occupational therapy $(N=1)$ and TAU $(N=5)(g=0.412,95 \%$ CI $0.19-0.64$, $p<0.001)$. Three interventions combined exercise with cognitive remediation.

An older meta-analysis [70] (AMSTAR $=4$, AMSTAR $+=2$ ) of people with serious mental illness (schizophrenia spectrum and bipolar disorder) suggested that exercise did not improve anxiety and depression scores combined (SMD $=-0.26,95 \% \mathrm{CI}-0.91$ to $0.39, \mathrm{RCT}=3$, $n=94, I^{2}=49 \%$ ). Six of the eight studies used TAU for their control condition.

\subsubsection{ADHD}

One meta-analysis [68] (AMSTAR $=8$, AMSTAR+=2) examined five trials of aerobic exercise versus usual care or education in children (mean age 11 years) diagnosed with ADHD. Overall exercise improved multiple outcomes including attention $(\mathrm{SMD}=0.84,95 \% \mathrm{CI} 0.48-1.20$, $\left.I^{2}=0 \%\right)$, hyperactivity $(\mathrm{SMD}=0.56,95 \%$ CI $0.04-1.08$, 
RCTs $\left.=2, n=62, I^{2}=0 \%\right)$, impulsivity $(\mathrm{SMD}=0.56$, $95 \%$ CI $0.04-1.08$, RCTs $=2, n=62, I^{2}=0 \%$ ), anxiety symptoms $(\mathrm{SMD}=0.66,95 \%$ CI $0.13-1.18, \mathrm{RCTs}=2$, $n=64, I^{2}=0 \%$ ), executive function (SMD $=0.58,95 \% \mathrm{CI}$ $0.15-1.00, \mathrm{RCTs}=3, n=102, I^{2}=8 \%$ ) and social disorders $\left(\mathrm{SMD}=0.59,95 \%\right.$ CI $\left.0.03-1.16, \mathrm{RCTs}=2, n=53, I^{2}=0 \%\right)$.

\subsection{Evidence for Exercise in Mental Disorders Without Meta-analyses of RCTs in Clinically Diagnosed Samples}

We did not find any meta-analyses that focussed on exercise RCTs with clinically diagnosed bipolar disorder, eating disorder, post-traumatic stress disorder, pre/post-natal depression, alcohol use disorders or substance use disorders. Thus, we identified 11 recent systematic reviews that have investigated the benefits of exercise in each condition, including in individuals symptomatic for these disorders (as identified by clinically validated tools). The summary of this evidence is presented in Table 2 .

\subsubsection{Bipolar Disorder}

A systematic review examined the association between exercise and bipolar disorder [71] across 31 studies $(n=15,587)$. No RCT was included and most studies were cross sectional. Exercise was found to be associated with less depressive symptoms and there was a lack of clarity with mania symptoms.

\subsubsection{Eating Disorders}

3.2.2.1 Anorexia Nervosa Moola et al. [72] conducted a systematic review of exercise in AN across five RCTs, two quasi-experimental studies, two case studies and one qualitative study. Participants ( $n$ exercise $=91 ; n$ control $=87$ ) were mostly female ( 7 males across all studies), with median age of 28 years (range of 11-45). The ten included studies varied in exercise type, with aerobic, anaerobic, and resistance training being used, with varied intensity and the duration lasting between 2 weeks and 12 months. The authors reported two studies which found that exercise reduced eating disorder symptoms (e.g. less food preoccupation) following exercise participation. No evidence of eating disorder distress was exacerbated by exercise [72]. Six studies found psychological wellbeing improved following participation in exercise. A separate systematic review, not included in the main results, reported that supervised exercise did not result in BMI or weight change in four RCTs [82], thus indicating a degree of safety.
3.2.2.2 Binge Eating Disorder Blanchet et al. [73] examined the effect of exercise on 842 people with binge eating disorder, across eight studies. The age range was $36-51$ years, mean BMI was $34 \mathrm{~kg} / \mathrm{m}^{2}$, and $84 \%$ of participants were women. Various types of exercise were used including home exercise practice, brisk walking and aerobic exercise. Among the RCTs, it was found that the number of BE episodes was significantly reduced with exercise compared to other interventions, and a significant decrease in depressive symptoms was also found when accompanied by the treatment of binge eating disorder. Two studies included cotreatments of CBT, and one study included a dietary group as a control condition; however, information regarding the other studies was not provided.

3.2.2.3 Bulimia Nervosa Vancampfort et al. [74] examined the effects of exercise in bulimia nervosa. One RCT focused on a once weekly, 60 min aerobic exercise program. In 64 women (age range $=18-29$ years, $\mathrm{BMI}=20.3 \mathrm{~kg} / \mathrm{m}^{2}$ ), exercisers reported 18 months post-intervention significantly less binges and vomiting compared to control interventions that included cognitive behavioural therapy and nutritional counselling. One study involved the use of Basic Body Awareness Therapy as a co-treatment. Control conditions included TAU, CBT, nutrition counselling and wait list.

\subsubsection{Post-traumatic Stress Disorder (PTSD)}

Rosenbaum et al. [75] examined the effect of exercise on PTSD in a systematic review of four RCTs $(n=200$, age range of 34-52 years). Although two of the included studies investigated the impact of yoga, data from two of the pooled trials utilising combined aerobic and resistance, and aerobiconly exercises, provided promising evidence that exercise can significantly reduce PTSD symptoms, compared to control conditions. Preliminary evidence also suggests that compared to controls, interventions with exercise are significantly more effective at decreasing depressive symptoms in people experiencing PTSD.

\subsubsection{Pre/Post-natal Depression}

A meta-analysis [76] examining the use of exercise in the prevention or treatment of postpartum depression included 17 RCTs $(n=1428)$ which involved aerobic exercise and coaching compared to usual care-non-intervention and active controls. Compared to control conditions, exercise was effective in reducing depressive symptoms $(\mathrm{SMD}=-0.64,95 \%$ $\mathrm{CI}-0.96$ to $-0.33, p<0.001)$. Control conditions included TAU, informal social support sessions and health education.

A meta-analysis of 12 RCTs ( $n$ exercise $=471, n$ control $=461$, TAU) examined the effect of exercise-based 


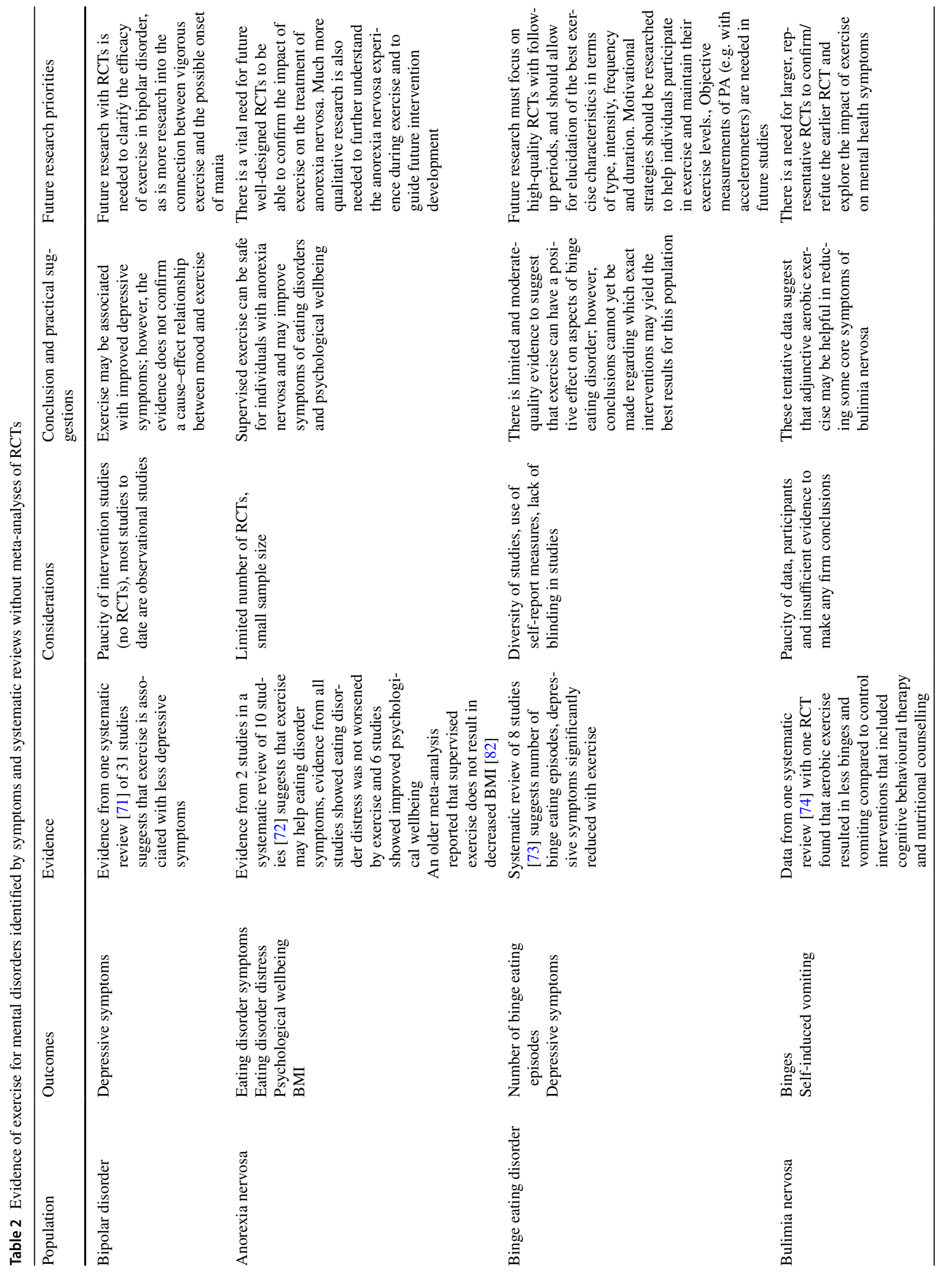




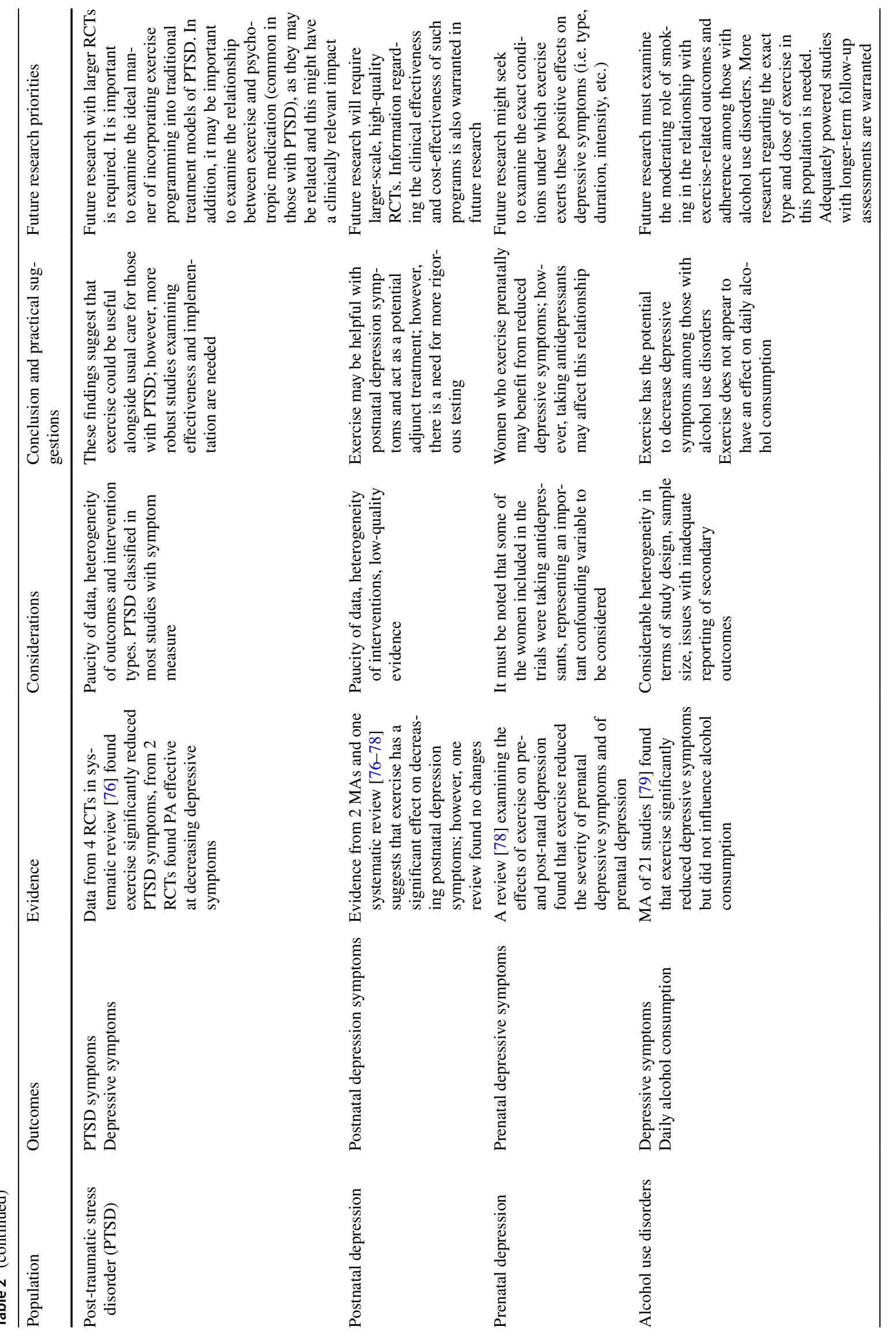


interventions on postpartum depressive symptoms [77]. Interventions varied and included both aerobic and resistance training, home-based programs and brisk walking programs, and sessions varied from one to five per week. Exercise interventions reduced postpartum depressive symptoms during pregnancy and the postpartum period $(\mathrm{SMD}=0.41$, 95\% CI 0.28-0.54).

A final review [78] examining the effect of prenatal exercise on depression and anxiety during both the pregnancy and the postpartum period included 52 studies $(n=131,406)$. Interventions ranged from 20 to 75 min per session, from 1 to 7 days/week, and included aerobic exercise, resistance training and pelvic floor muscle training. Co-treatments included diet, education classes and a smoking cessation program, along with exercise. Evidence from RCTs found that exercise interventions, compared to no exercise, decreased the severity of prenatal depressive symptoms (13 RCTs, $n=1076$; SMD $=-0.38,95 \% \mathrm{CI}-0.51$ to -0.25 , $I^{2}=10 \%$ ), and reduced the odds of prenatal depression by $67 \%$ (5 RCTs, $n=683$; OR $0.33,95 \%$ CI $0.21-0.53, I^{2}=0 \%$ ).

\subsubsection{Alcohol Use Disorder/Any Substance Use Disorder}

Hallgren et al. [79] examined the use of exercise as treatment for alcohol use disorders among 21 studies $(n=1204$ people, 37.8 years, illness duration of 4.4 years). The mean duration of exercise session was $43 \mathrm{~min}(\mathrm{SD}=19 \mathrm{~min})$ and interventions involved aerobic exercise and strength training. Whilst exercise did not reduce daily alcohol consumption or total scores on the Alcohol Use Disorders Identification Test, it reduced depressive symptoms when compared to controls $\left(\mathrm{RCTs}=4 ; \mathrm{SMD}=-0.867, p=0.006, I^{2}=63 \%\right)$.

Among individuals with multiple substance use disorders, Colledge et al. [80] examined the effect of anaerobic exercise specifically, in a systematic review of 26 studies (nicotine dependence $=12$; alcohol dependence $=1$; illicit drug dependence $=13$ ). The mean sample size was 97 , with a mean age of 34.3 years. With the exception of four studies investigating acute exercise bouts, all others examined long-term interventions, with varying levels of intensity. The results of the study were extremely mixed; however, some positive effects (not significant) were found for the outcome of abstinence in nicotine dependence. Control conditions included TAU, health education and behavioural modification training.

In a separate meta-analysis among individuals with substance use disorders, 22 studies were included to examine whether physical exercise could act as a treatment for substance use disorders, with the primary outcome being abstinence rates [81]. Exercises varied from light-vigorous intensity and included aerobic and some mind-body exercises. When individuals of all substance use disorders were grouped together (i.e. nicotine, alcohol, illicit drug users), it 
was found that exercise effectively increased abstinence rates $\mathrm{OR}=1.69$ (95\% CI 1.44, 1.99), eased withdrawal symptoms $\mathrm{SMD}=-1.24(95 \% \mathrm{CI}-2.46,-0.02)$, and reduced symptoms of anxiety SMD $=-0.31(95 \% \mathrm{CI}-0.45,-0.16)$ and depression $\mathrm{SMD}=-0.47(95 \% \mathrm{CI}-0.80,-0.14)$.

\section{Discussion}

Our meta-review examined the potential benefits of exercise for neuropsychiatric and cognitive symptoms across the spectrum of mental disorders. By combining evidence from 27 different systematic reviews and meta-analyses, we produced a number of novel findings. First, we found relatively consistent evidence across eight meta-analyses [57-64] that structured moderate-to-vigorous intensity exercise can have a positive impact on symptoms of depression as an add-on treatment in adolescents, working age and older adults. Second, there is tentative evidence that moderate-to-vigorous intensity aerobic and resistance training exercise improves mental health symptoms and cognition in people with schizophrenia spectrum disorders $[33,34,70]$. Third, the data indicate that moderate-to-vigorous intensity aerobic exercise can have a positive impact on multiple outcomes in children with ADHD [68]. Fourth, in people with PTSD (as indicated by validated measures, but without confirmed diagnoses), preliminary evidence suggests that moderate-to-vigorous intensity exercise can reduce symptoms of both PTSD and depression [75]. There is also good evidence that moderateto-vigorous intensity exercise in the pre- and postpartum period can reduce depressive symptoms and the odds of developing depression [76-78]. Among people with alcohol use disorder, preliminary evidence suggests that moderate-to-vigorous intensity exercise may improve depressive symptoms but does not appear to influence alcohol intake [79], although this evidence was based on three trials. Available evidence indicates that moderate-to-vigorous intensity exercise may improve SUD abstinence rates and anxiety/ depressive symptoms across multiple SUDs [80, 81]. There is some tentative evidence that supervised exercise may be helpful for people with AN [72] without negatively impacting BMI [82] and may help improve ED symptoms in bulimia nervosa [72] whilst also potentially reducing binge eating disorder symptomology and depressive symptoms and improving psychological wellbeing in binge eating disorders [71]. Finally, limited data from interventions are available to support the use of moderate-to-vigorous intensity exercise in bipolar disorder, although observational data imply that exercise can have a positive impact on depressive symptomology. Taken together, our data provide robust evidence for the value of moderate-to-vigorous intensity exercise as a potential transdiagnostic intervention for mental health symptoms across people with mental disorders.

\subsection{Potential Mechanisms}

Despite the increasing evidence base for the positive effects of exercise on mental health symptoms in people with mental disorders, relatively little has been established in humans about the underlying mechanisms. This lack of clarity is perhaps exemplified by the most densely researched area of exercise and MDD where a recent systematic review [83] found tentative evidence that acute (i.e. a single bout) exercise may exert its antidepressant effect by increasing atrial natriuretic peptide, brain natriuretic peptide, copeptin and growth hormone among people with MDD. The review [83] also found that longer-term exercise may exert its antidepressant effect by promoting long-term adaptations of copeptin, thiobarbituric acid reactive species and total mean frequency. A recent acute exercise study [84] and longer-term RCT [85] demonstrated that aerobic exercise may exert its effect by increasing brain-derived neurotrophic factors (BDNF). However, a preliminary meta-analysis [86] of only 6 RCTs and 176 participants found that longer-term changes in BDNF fell short of significance (SMD $=0.43,95 \%$ CI $-0.06-0.92$, $p=0.09$ ). Among people with schizophrenia, the potential positive effects on mental health and cognitive symptoms have been suggested to be related to increases in hippocampal volume [87], yet these findings in mental disorders have not been replicated in a recent meta-analysis of RCTs [8]. Nonetheless, neurocognitive improvements following exercise in schizophrenia may again be linked to increased levels of BDNF [32]. However, psychosocial mechanisms also play a pivotal role in the mental health benefits of exercise, such as increased social support and reduced social isolation [88], improved self-esteem [89] and body image [90]. Among people with anxiety and stress disorders, a number of theories with modest amounts of data have suggested that increasing self-esteem, adaptations in GABA levels, adaptations in norepinephrine and the serotonin neurotransmitter system may account for the anxiolytic effects [91].

\subsection{Exercise and Physical Health}

Whilst not the focus of this review, along with the neuropsychiatric benefits, exercise interventions can also play an important role in reducing the physical health inequalities observed in people with mental illness [44]. Specifically, there is a plethora of evidence from the general population that exercise can reduce the risk of cardio-metabolic diseases (such as obesity, diabetes and metabolic syndrome $[92,93])$. This is particularly relevant to psychiatric populations, as people with mental illness are significantly less active and more sedentary than the general population [94], and this has been identified as a transdiagnostic risk factor for the elevated cardio-metabolic risk [95-97]. Moreover, increased cardio-metabolic dysfunction has been associated 
with worse mental and cognitive symptoms in those with schizophrenia [98], MDD [99], bipolar disorder [100] and binge eating disorder [101]. Clearly, exercise has considerable potential to act as a transdiagnostic "polypill" across multiple domains (mental, cognitive, physical) and future ambitious well-powered RCTs are required to target these multiple domains affected by people with mental disorders.

\subsection{Exercise and Depressive Disorders}

To date, most evidence for exercise interventions in psychiatry is for depressive disorders-where there is relative harmony across the seven meta-analyses of RCTs that exercise is an effective add-on treatment to usual care to reduce symptoms of depression in adolescents [57, 58], working age [60-63] and older adults [59]. There is also some tentative evidence from comparative meta-analyses that exercise has similar effects to psychotherapy [61] and antidepressant medications [61, 62]. However, caution should be taken in the interpretation of these comparisons, due to the small number of participants (typically $<300$ ) and trials $(<5)$ in such analyses. A previous Cochrane review [64] found that exercise was not effective in reducing depressive symptoms in a subgroup of "high-quality" studies that included subthreshold depression. This Cochrane review [64] was heavily criticised for multiple methodological and selection biases [102]. Subsequent meta-analyses in working age adults [60, $61,63]$ have consistently reported the overall benefits of exercise but reported equivocal data regarding the outcome in "high-quality studies". For instance, Schuch et al. [60] found that exercise was effective in high-quality RCTs when considering those with MDD and subthreshold depressive symptoms but not in MDD only (although limited to 2 RCTs). Kvam et al. [61] and another meta-analysis [103] published after our search date found that aerobic exercise was more effective than control conditions for depressive symptoms in MDD in RCTs at low risk of bias. However, a further trial sequential meta-analysis [63] of four "highquality" RCTs which compared exercise versus other active interventions (including PA) found no effect. Clearly, whilst there is a consensus of the benefit of exercise for depression, there is a need for larger and better controlled RCTs to be developed in MDD to attempt to match the standards in other areas of psychiatry such as psychological therapy.

For optimal outcomes in MDD, it appears that supervised exercise interventions tailored to the individual have larger effect sizes [60]. A major criticism of the potential benefits of exercise for MDD is the relatively short follow-up of most studies ( $<6$ months). Whilst the diagnoses of depression were identified through the Patient Health Questionnaire [104], a recent large-scale $(n=945)$ study [105] has partly addressed this concern, finding that aerobic exercise was equally as effective as internet delivered CBT and better than usual care after 12 months. Whilst there is some encouraging evidence of the role of resistance training alone in people with depressive symptoms [106], there are very limited data available to date on exercise as a stand-alone intervention in people with MDD. Thus, moderate-to-vigorous intensity aerobic exercise alone or in combination with resistance training [44] over at least 12 weeks and achieving $90 \mathrm{~min}$ per week appears the optimal mode of delivery of structured exercise. Whilst this is informed by the evidence base in people with MDD, one should note that people should be encouraged to adopt general guidelines of $150 \mathrm{~min}$ of vigorous PA per week [23, 43].

Among people with MDD, one meta-analysis [64] found that aerobic exercise had no main effects on cognitive functioning. However, almost all the included studies did not set out with the primary objective to influence cognition and no studies included people with cognitive dysfunction at baseline. Whilst there is robust evidence in the general population for the potential of exercise for preserving and improving cognition [11, 12, 107], future adequately powered RCTs with the primary objective of improving cognition are needed. Future interventions should also examine the role of resistance training for improving cognition in serious mental illness, since there is a clear association between increased muscular strength and cognition in people with major depression, bipolar disorder and schizophrenia $[108,109]$ and evidence from the general population indicates resistance training can improve cognition $[110,111]$.

\subsection{Exercise and Schizophrenia/Serious Mental Illness}

Four meta-analyses investigated exercise in people with schizophrenia/serious mental illness [33, 34, 80, 81]. Whilst the data are encouraging for core psychiatric symptoms [34, 69], this finding requires replication in large, controlled trials. Nonetheless, the emerging data suggest that higher intensities of aerobic exercise may produce neuropsychiatric benefits in this population $[33,34,80]$. For instance, for cognitive outcomes, greater effect sizes were observed by interventions which administered higher doses of exercise (minutes per week) and were delivered by a qualified exercise professional [33]. Similarly, significant effects on positive and negative symptoms were only observed in RCTs which administered at least 90 min per week of moderateto-vigorous intensity exercise (with no symptomatic benefits observed from low-intensity training) [34]. It does appear from the paucity of RCTs available that aerobic exercise does improve depressive symptoms in schizophrenia [69]. Depression is highly comorbid in schizophrenia and treatment options are limited [112]; thus, future well-powered RCTs are required that specifically include people with schizophrenia and depression to elucidate if exercise can 
improve depressive symptoms. In schizophrenia/serious mental illness, adverse event reporting at a meta-analytical level is sparse, whilst dropout from exercise interventions appears higher than in control groups [113]. Given the mental health benefits, along with the clear potential for exercise to improve the poor physical health among people with schizophrenia [44], further research should now be dedicated towards establishing feasible and sustainable methods for engaging this population with moderate-to-vigorous intensity exercise training in real-world settings.

\subsection{Exercise and Stress Disorders}

In those with anxiety and stress disorders, there was evidence that exercise was effective versus non-active interventions in reducing anxiety symptoms [33, 34, 69]. The RCTs in anxiety and stress disorders to date have included relatively few people and there are a small number of RCTs, clearly emphasising a need for larger-scaled RCTs. To date, most RCTs have focussed on aerobic exercise in people with clinical anxiety/stress disorders. However, there is encouraging evidence of the benefits of resistance training for people with elevated symptoms of anxiety [115] and testing resistance training protocols in those with anxiety/stress disorders should be a future priority.

\subsection{Exercise and ADHD}

Despite the small number of participants and RCTs, there is also encouraging evidence that aerobic exercise can improve multiple cognitive domains in children with ADHD [68]. Similar positive effects of the benefits of aerobic exercise on cognition, mental health and academic achievement have been observed in children in the general population $[5,6,115]$. Given the burden of attention, behavioural and cognitive deficits often noted in children with ADHD and concerns about over prescription of medications with side effects [116], exercise could hold promise as an adjunctive treatment and requires testing in robust well-powered RCTs.

\subsection{Exercise and Mental Health Symptoms}

Among people with mental disorders identified through clinically validated measures (but in absence of confirmed diagnoses), there is encouraging evidence for improved mental health outcomes in those with PTSD [75], anorexia nervosa [72], bulimia nervosa [74], binge eating disorder [73], alcohol use disorder [79], substance use disorders [80, 81] and pre/post-natal depression [76-78]. With the exception of the pre- and post-natal depression reviews [76-78], the sample sizes were relatively small in these areas and future adequately powered RCTs are needed to confirm/refute the encouraging work in these areas. Disappointingly, there is a dearth of evidence from RCTs that can elucidate the potential benefits of exercise for mental health or cognitive symptoms in people with bipolar disorder.

\subsection{Strengths and Limitations}

Whilst this meta-review provides a comprehensive overview of exercise for all mental and SUDs, a number of limitations prevail. First, there was considerable heterogeneity in the exercise interventions across the included systematic reviews and specific details regarding the frequency, intensity, type and time of exercise were often lacking. This rendered it impossible to make meaningful direct statistical comparisons between different modes of exercise (e.g. resistance versus aerobic exercise) within and across mental disorders. Future research should thus clearly outline exercise protocols using recognised guidelines (e.g. [117]) to maximise the potential translation into clinical practice. Second, for many of the mental disorder categories, the number of RCTs and included participants was small and included samples that are unrepresentative of the realities of clinical practice. To overcome this, future research could consider examining the benefits of exercise randomised at a service level. One option to assess this would be using cluster RCTs to randomly allocate clinical services to either exercise interventions (active) or control conditions and then assess the extent to which integration of exercise interventions within services improves the patient outcomes, compared to usual service provision. Such studies could also be used to assess the costeffectiveness of delivering exercise interventions within psychiatric care. Another methodological approach is to use multiple baseline or stepped-wedged designs in which individual participants can act as their own control to overcome the ethical issues associated with delaying or withholding access to evidence-based exercise interventions. Third, there is a relative paucity of data on the impact of exercise in adolescents and youth with mental disorders. This is particularly relevant given the concerns about adverse outcomes with pharmacotherapy in youth $[30,118]$. Fourth, based on the current AMSTAR+ scores publication bias was potentially problematic for the majority of the meta-analyses, potentially overestimating the pooled effect sizes. Fifth, owing to the complex nature of the field, there was variation in the calculation and reporting of effect sizes, publication bias, heterogeneity and general reporting. This makes comparison across the field at times challenging and calls for some standardisation in the future. Finally, there is a paucity of data on potential adverse events and cost-effectiveness data from exercise interventions. However, several recent studies have suggested that exercise is safe in serious mental illness $[44,119]$. Future long-term research is required to address this important oversight. 
Despite these aforementioned limitations, the field of exercise science has in the space of 20 years made significant strides in establishing itself as an important adjunctive treatment for mental disorders, underpinned by ever increasing robust science. Whilst the focus of exercise in psychiatric multidisciplinary teams has revolved around the need to address the unanimous poor physical health across mental disorders [44], our review adds support to the utilisation of exercise as a treatment for neuropsychiatric symptoms. Beyond MDD, we are not yet at the stage of making evidence-based bespoke recommendations on the optimal clinical delivery of exercise. Thus, psychiatric teams should seek to follow recent exercise guidelines which emphasise the need for people to be supported to achieve 150-300 $\mathrm{min}$ or 75-150 min of moderate or vigorous exercise over each week [24]. Given the unanimous low levels of moderatevigorous PA in mental disorders $([94,120-122]$, with the exception of AN [123]), achieving such targets is aspirational. Thus, people should be encouraged to start making small changes to increase their exercise levels, with the support of recognised exercise professionals. In addition, recent guidelines recommend that resistance training be undertaken twice a week [24]. There is encouraging evidence for resistance training in people with elevated mental health symptoms [108-114], but this requires further testing in those with mental disorders. Beyond treatment, there are encouraging data considering the potential for exercise to act as a primary prevention for mental disorders that should be tested further. There are recent large-scale observational [18] and Mendelian randomisation studies [124] suggesting that higher levels of PA can confer protection against mental disorders. However, this has yet to be tested in experimental studies, and so the key question remains as to whether increasing an individual's PA behaviour can actually prevent the onset of psychiatric conditions. These data have significant implications for public health campaigns targeting PA which should ensure that the mental health benefits are appropriately prioritised alongside the physical health benefits.

\section{Conclusion}

In conclusion, in those with mental disorders, there is promising evidence that exercise has a positive impact on mental health symptoms and tentative evidence for benefits for cognition in schizophrenia spectrum disorders. There is consistent evidence that exercise can reduce depressive symptoms in children, adults and older adults with encouraging evidence in anxiety and stress disorders, children with ADHD and people with schizophrenia spectrum. Among people with elevated mental health symptoms, exercise can improve multiple mental health outcomes in those with anorexia nervosa, binge eating disorder, bulimia nervosa, alcohol use disorders, substance use disorders and pre- and post-natal depression; however, further research is needed in these conditions. Given these findings, the potential for exercise to act as a transdiagnostic treatment is considerable. Future research should prioritise robust RCTs that can directly inform the design and delivery of exercise interventions across a range of settings (including low resource settings), diagnoses and demographics to ensure the translation of this body of evidence into routine clinical practice.

Author contributions BS, JF and DV designed the review. BS, AK, MH, JF, FS, GA-F, MS, SR and RC conducted the literature searches and extracted the data. BS, JF, DV and GA-F wrote the paper. All authors provided critical comments, approved the final version and meet the criteria for authorship.

Data Availability Statement As this article is a review, the data can be found within each article referenced (see References list below).

\section{Compliance with Ethical Standards}

Funding Brendon Stubbs holds a Clinical Lectureship supported by Health Education England and the NIHR Integrated Clinical Academic (ICA) Programme (ICA-CL-2017-03-001). Brendon Stubbs is also part supported by the Maudsley Charity and the National Institute for Health Research (NIHR) Collaboration for Leadership in Applied Health Research and Care South London at King's College Hospital NHS Foundation Trust. The views expressed are those of the author[s] and not necessarily those of the NHS, the NIHR or the Department of Health and Social Care. John Firth is supported by a Blackmores Institute Fellowship. Rebekah Carney is funded by the Research Capability Fund via Greater Manchester West Mental Health NHS Foundation Trust. Garcia Ashdown-Franks is funded by a Mitacs Globalink Research Award.

Conflict of interest Garcia Ashdown-Franks, Joseph Firth, Rebekah Carney, Andre Carvalho, Mats Hallgren, Ai Koyanagi, Simon Rosenbaum, Felipe Schuch, Lee Smith, Marco Solmi, Davy Vancampfort and Brendon Stubbs declare that they have no conflicts of interest relevant to the content of this review.

\section{References}

1. Caspersen CJ, Powell KE, Christenson GM. Physical activity, exercise, and physical fitness: definitions and distinctions for health-related research. Public Health Rep (Washington, DC: 1974). 1985;100(2):126-31.

2. Daskalopoulou C, Stubbs B, Kralj C, Koukounari A, Prince M, Prina AM. Physical activity and healthy ageing: a systematic review and meta-analysis of longitudinal cohort studies. Ageing Res Rev. 2017;38:6-17.

3. Kelley GA, Kelley KS. Exercise and sleep: a systematic review of previous meta-analyses. J Evid Based Med. 2017;10(1):26-36.

4. Lederman O, Ward PB, Firth J, et al. Does exercise improve sleep quality in individuals with mental illness? A systematic review and meta-analysis. J Psychiatr Res. 2019;109:96-106.

5. Lubans D, Richards J, Hillman C, et al. Physical activity for cognitive and mental health in youth: a systematic review of mechanisms. Pediatrics. 2016;138(3):e20161642. 
6. Lubans D, Plotnikoff R, Lubans N. A systematic review of the impact of physical activity programmes on social and emotional well-being in at-risk youth. Child Adolesc Ment Health. 2011;17(1):2-13.

7. Hamer M, Chida Y. Physical activity and risk of neurodegenerative disease: a systematic review of prospective evidence. Psychol Med. 2009;39(1):3-11.

8. Firth J, Stubbs B, Vancampfort D, et al. Effect of aerobic exercise on hippocampal volume in humans: a systematic review and meta-analysis. Neuroimage. 2018;166:230-8.

9. Voss MW, Erickson KI, Prakash RS, et al. Neurobiological markers of exercise-related brain plasticity in older adults. Brain Behav Immun. 2013;28:90-9.

10. Voss MW, Vivar C, Kramer AF, van Praag H. Bridging animal and human models of exercise-induced brain plasticity. Trends Cogn Sci. 2013;17(10):525-44.

11. Prakash RS, Voss MW, Erickson KI, Kramer AF. Physical activity and cognitive vitality. Annu Rev Psychol. 2015;66(1):769-97.

12. Kramer AF, Erickson KI. Capitalizing on cortical plasticity: influence of physical activity on cognition and brain function. Trends Cogn Sci. 2007;11(8):342-8.

13. Blondell SJ, Hammersley-Mather R, Lennert Veerman J. Does physical activity prevent cognitive decline and dementia? A systematic review and meta-analysis of longitudinal studies. BMC Public Health. 2014;14(1):1036-61.

14. Erickson KI, Voss MW, Prakash RS, et al. Exercise training increases size of hippocampus and improves memory. Proc Natl Acad Sci USA. 2011;108(7):3017-22.

15. Poitras VJ, Gray CE, Borghese MM, et al. Systematic review of the relationships between objectively measured physical activity and health indicators in school-aged children and youth. Appl Physiol Nutr Metab. 2016;41(6 Suppl 3):S197-239.

16. Hamer M, Stamatakis E, Steptoe A. Dose-response relationship between physical activity and mental health: the Scottish health survey. Br J Sports Med. 2009;43(14):1111-4.

17. Biddle SJ, Asare M. Physical activity and mental health in children and adolescents: a review of reviews. Br J Sports Med. 2011;45(11):886-95.

18. Schuch FB, Vancampfort D, Firth J, et al. Physical activity and incident depression: a meta-analysis of prospective cohort studies. Am J Psychiatry. 2018:appiajp201817111194.

19. Harvey SB, Overland S, Hatch SL, Wessely S, Mykletun A, Hotopf M. Exercise and the prevention of depression: results of the HUNT cohort study. Am J Psychiatry. 2017:appiajp201716111223.

20. McDowell CP, Dishman RK, Vancampfort D, et al. Physical activity and generalized anxiety disorder: results from The Irish Longitudinal Study on Ageing (TILDA). Int J Epidemiol. 2018;47(5):1443-53

21. LeardMann CA, Kelton ML, Smith B, et al. Prospectively assessed posttraumatic stress disorder and associated physical activity. Public Health Rep. 2011;126(3):371-83.

22. Chastin SFM, De Craemer M, De Cocker K, et al. How does light-intensity physical activity associate with adult cardiometabolic health and mortality? Systematic review with meta-analysis of experimental and observational studies. Br J Sports Med. 2018;53(6):370-6.

23. Nelson ME, Rejeski WJ, Blair SN, et al. Physical activity and public health in older adults: recommendation from the American College of Sports Medicine and the American Heart Association. Med Sci Sports Exerc. 2007;39(8):1435-45.

24. Piercy KL, Troiano RP, Ballard RM, et al. The physical activity guidelines for Americans. JAMA. 2018;320(19):2020-8.

25. Vancampfort D, Firth J, Correll CU, et al. The impact of pharmacological and non-pharmacological interventions to improve physical health outcomes in people with schizophrenia: a meta-review of meta-analyses of randomized controlled trials. World Psychiatry. 2019;18(1):53-66.

26. Vancampfort D, Correll CU, Galling B, et al. Diabetes mellitus in people with schizophrenia, bipolar disorder and major depressive disorder: a systematic review and large scale meta-analysis. World Psychiatry. 2016;15(2):166-74.

27. Vancampfort D, Stubbs B, Mitchell AJ, et al. Risk of metabolic syndrome and its components in people with schizophrenia and related psychotic disorders, bipolar disorder and major depressive disorder: a systematic review and meta-analysis. World Psychiatry. 2015;14(3):339-47.

28. Correll CU, Solmi M, Veronese N, et al. Prevalence, incidence and mortality from cardiovascular disease in patients with pooled and specific severe mental illness: a large-scale meta-analysis of $3,211,768$ patients and 113,383,368 controls. World Psychiatry. 2017;16(2):163-80.

29. Solmi M, Veronese N, Correll CU, et al. Bone mineral density, osteoporosis, and fractures among people with eating disorders: a systematic review and meta-analysis. Acta Psychiatr Scand. 2016;133(5):341-51.

30. Galling B, Roldan A, Nielsen RE, et al. Type 2 diabetes mellitus in youth exposed to antipsychotics: a systematic review and meta-analysis. JAMA Psychiatry. 2016;73(3):247-59.

31. Arcelus J, Mitchell AJ, Wales J, Nielsen S. Mortality rates in patients with anorexia nervosa and other eating disorders. A meta-analysis of 36 studies. Arch Gen Psychiatry. 2011;68(7):724-31.

32. Firth J, Cotter J, Carney R, Yung AR. The pro-cognitive mechanisms of physical exercise in people with schizophrenia. Br J Pharmacol. 2017;174(19):3161-72.

33. Firth J, Stubbs B, Rosenbaum S, et al. Aerobic exercise improves cognitive functioning in people with schizophrenia: a systematic review and meta-analysis. Schizophr Bull. 2016;43(3):546-56.

34. Firth J, Cotter J, Elliott R, French P, Yung AR. A systematic review and meta-analysis of exercise interventions in schizophrenia patients. Psychol Med. 2015;45(7):1343-61.

35. Correll CU, Rubio JM, Kane JM. What is the risk-benefit ratio of long-term antipsychotic treatment in people with schizophrenia? World Psychiatry. 2018;17(2):149-60.

36. Jorm AF, Patten SB, Brugha TS, Mojtabai R. Has increased provision of treatment reduced the prevalence of common mental disorders? Review of the evidence from four countries. World Psychiatry. 2017;16(1):90-9.

37. Cuijpers P, Cristea IA, Karyotaki E, Reijnders M, Huibers MJ. How effective are cognitive behavior therapies for major depression and anxiety disorders? A meta-analytic update of the evidence. World Psychiatry. 2016;15(3):245-58.

38. Bighelli I, Salanti G, Huhn M, et al. Psychological interventions to reduce positive symptoms in schizophrenia: systematic review and network meta-analysis. World Psychiatry. 2018;17(3):316-29.

39. Kahn RS, Keefe RS. Schizophrenia is a cognitive illness: time for a change in focus. JAMA Psychiatry. 2013;70(10):1107-12.

40. Elias LR, Miskowiak KW, Vale AM, et al. Cognitive impairment in euthymic pediatric bipolar disorder: a systematic review and meta-analysis. J Am Acad Child Adolesc Psychiatry. 2017;56(4):286-96.

41. Bortolato B, Miskowiak KW, Kohler CA, Vieta E, Carvalho AF. Cognitive dysfunction in bipolar disorder and schizophrenia: a systematic review of meta-analyses. Neuropsychiatr Dis Treat. 2015;11:3111-25.

42. Bortolato B, Carvalho AF, McIntyre RS. Cognitive dysfunction in major depressive disorder: a state-of-the-art clinical review. CNS Neurol Disord Drug Targets. 2014;13(10):1804-18. 
43. Hirst RB, Beard CL, Colby KA, Quittner Z, Mills BM, Lavender JM. Anorexia nervosa and bulimia nervosa: a meta-analysis of executive functioning. Neurosci Biobehav Rev. 2017;83:678-90.

44. Stubbs B, Vancampfort D, Hallgren M, et al. EPA guidance on physical activity as a treatment for severe mental illness: a meta-review of the evidence and Position Statement from the European Psychiatric Association (EPA), supported by the International Organization of Physical Therapists in Mental Health (IOPTMH). Eur Psychiatry. 2018;54:124-44.

45. Rosenbaum S, Hobson-Powell A, Davison K, Stanton R, Craft LL, Duncan M, Elliot C, Ward PB. The role of sport, exercise, and physical activity in closing the life expectancy gap for people with mental illness: an international consensus statement by Exercise and Sports Science Australia, American College of Sports Medicine, British Association of Sport and Exercise Science, and Sport and Exercise Science New Zealand. Transl J Am Coll Sports Med. 2018;3(10):72-3.

46. American Psychiatric Association. Diagnostic and statistical manual of mental disorders-DSM-IV-TR. 4th edition. In: American Psychiatric Association; 2000.

47. World Health Organisation. The ICD-10 classification of mental and behavioural disorders-Diagnostic criteria for research. 1993.

48. Bridle C, Spanjers K, Patel S, Atherton NM, Lamb SE. Effect of exercise on depression severity in older people: systematic review and meta-analysis of randomised controlled trials. Br J Psychiatry J Ment Sci. 2012;201(3):180-5.

49. Nyer M, Nauphal M, Roberg R, Streeter C. Applications of yoga in psychiatry: what we know. FOCUS. 2018;16(1):12-8.

50. Higgins JPT, Thompson SG, Deeks JJ, Altman DG. Measuring inconsistency in meta-analyses. BMJ. 2003;327(7414):557-60.

51. Higgins JPT, Green S. Cochrane handbook for systematic reviews of interventions version 5.1.0. In. The Cochrane Collaboration. 2011. http://www.cochrane-handbook.org. Accessed Mar 2011.

52. Egger M, Davey Smith G, Schneider M, Minder C. Bias in metaanalysis detected by a simple, graphical test. BMJ (Clin Res Ed). 1997;315(7109):629-34.

53. Shea BJ, Hamel C, Wells GA, et al. AMSTAR is a reliable and valid measurement tool to assess the methodological quality of systematic reviews. J Clin Epidemiol. 2009;62(10):1013-20.

54. Shea BJ, Grimshaw JM, Wells GA, et al. Development of AMSTAR: a measurement tool to assess the methodological quality of systematic reviews. BMC Med Res Methodol. 2007;7:10-7.

55. Sharif MO, Janjua-Sharif FN, Ali H, Ahmed F. Systematic reviews explained: AMSTAR-how to tell the good from the bad and the ugly. Oral Health Dent Manag. 2013;12(1):9-16.

56. Correll CU, Rubio JM, Inczedy-Farkas G, Birnbaum ML, Kane JM, Leucht S. Efficacy of 42 Pharmacologic cotreatment strategies added to antipsychotic monotherapy in schizophrenia: systematic overview and quality appraisal of the aeta-analytic evidence. JAMA Psychiatry. 2017;74(7):675-84.

57. Bailey AP, Hetrick SE, Rosenbaum S, Purcell R, Parker AG. Treating depression with physical activity in adolescents and young adults: a systematic review and meta-analysis of randomised controlled trials. Psychol Med. 2018;48(7):1068-83.

58. Carter T, Morres ID, Meade O, Callaghan P. The effect of exercise on depressive symptoms in adolescents: a systematic review and meta-analysis. J Am Acad Child Adolesc Psychiatry. 2016;55(7):580-90.

59. Schuch FB, Vancampfort D, Rosenbaum S, et al. Exercise for depression in older adults: a meta-analysis of randomized controlled trials adjusting for publication bias. Rev Bras Psiquiatr. 2016;38(3):247-54.
60. Schuch FB, Vancampfort D, Richards J, Rosenbaum S, Ward PB, Stubbs B. Exercise as a treatment for depression: a meta-analysis adjusting for publication bias. J Psychiatr Res. 2016;77:42-51.

61. Kvam S, Kleppe CL, Nordhus IH, Hovland A. Exercise as a treatment for depression: a meta-analysis. J Affect Disord. 2016;202:67-86.

62. Cooney GM, Dwan K, Greig CA, et al. Exercise for depression. Cochrane Database Syst Rev. 2013;9:CD004366.

63. Krogh J, Hjorthoj C, Speyer H, Gluud C, Nordentoft M. Exercise for patients with major depression: a systematic review with meta-analysis and trial sequential analysis. BMJ Open. 2017;7(9):e014820.

64. Brondino N, Rocchetti M, Fusar-Poli L, et al. A systematic review of cognitive effects of exercise in depression. Acta Psychiatr Scand. 2017;135(4):285-95.

65. Stubbs B, Vancampfort D, Rosenbaum S, et al. An examination of the anxiolytic effects of exercise for people with anxiety and stress-related disorders: a meta-analysis. Psychiatry Res. 2017;249:102-8.

66. Bartley CA, Hay M, Bloch MH. Meta-analysis: aerobic exercise for the treatment of anxiety disorders. Prog Neuropsychopharmacol Biol Psychiatry. 2013;45:34-9.

67. Aylett E, Small N, Bower P. Exercise in the treatment of clinical anxiety in general practice-a systematic review and metaanalysis. BMC Health Serv Res. 2018;18(1):559.

68. Cerrillo-Urbina AJ, Garcia-Hermoso A, Sanchez-Lopez M, Pardo-Guijarro MJ, Santos Gomez JL, Martinez-Vizcaino V. The effects of physical exercise in children with attention deficit hyperactivity disorder: a systematic review and metaanalysis of randomized control trials. Child Care Health Dev. 2015;41(6):779-88.

69. Dauwan M, Begemann MJ, Heringa SM, Sommer IE. Exercise improves clinical symptoms, quality of life, global functioning, and depression in schizophrenia: a systematic review and metaanalysis. Schizophr Bull. 2016;42(3):588-99.

70. Pearsall R, Smith DJ, Pelosi A, Geddes J. Exercise therapy in adults with serious mental illness: a systematic review and metaanalysis. BMC Psychiatry. 2014;14(1):1-28.

71. Melo MC, Daher Ede F, Albuquerque SG, de Bruin VM. Exercise in bipolar patients: a systematic review. J Affect Disord. 2016;198:32-8.

72. Moola FJGS, Amara CE. Exercise in the care of patients with anorexia nervosa: a systematic review of the literature. Ment Health Phys Act. 2013;6(2):59-68.

73. Blanchet C, Mathieu ME, St-Laurent A, Fecteau S, St-Amour $\mathrm{N}$, Drapeau V. A systematic review of physical activity interventions in individuals with binge eating disorders. Curr Obes Rep. 2018;7(1):76-88.

74. Vancampfort D, Vanderlinden J, De Hert M, et al. A systematic review of physical therapy interventions for patients with anorexia and bulemia nervosa. Disabil Rehabil. 2014;36(8):628-34.

75. Rosenbaum S, Vancampfort D, Steel Z, Newby J, Ward PB, Stubbs B. Physical activity in the treatment of Post-traumatic stress disorder: a systematic review and meta-analysis. Psychiatry Res. 2015;230(2):130-6.

76. Carter T, Bastounis A, Guo B, Jane Morrell C. The effectiveness of exercise-based interventions for preventing or treating postpartum depression: a systematic review and meta-analysis. Arch Womens Ment Health. 2018;22:37-53.

77. Poyatos-Leon R, Garcia-Hermoso A, Sanabria-Martinez G, Alvarez-Bueno C, Cavero-Redondo I, Martinez-Vizcaino V. Effects of exercise-based interventions on postpartum depression: a meta-analysis of randomized controlled trials. Birth. 2017;44(3):200-8.

78. Davenport MH, McCurdy AP, Mottola MF, et al. Impact of prenatal exercise on both prenatal and postnatal anxiety and 
depressive symptoms: a systematic review and meta-analysis. Br J Sports Med. 2018;52(21):1376-85.

79. Hallgren M, Vancampfort D, Giesen ES, Lundin A, Stubbs B. Exercise as treatment for alcohol use disorders: systematic review and meta-analysis. Br J Sports Med. 2017;51(14):1058-64.

80. Colledge F, Gerber M, Puhse U, Ludyga S. Anaerobic exercise training in the therapy of substance use disorders: a systematic review. Front Psychiatry. 2018;9:644.

81. Wang D, Wang Y, Li R, Zhou C. Impact of physical exercise on substance use disorders: a meta-analysis. PLoS One. 2014;9(10):e110728.

82. Ng LW, Ng DP, Wong WP. Is supervised exercise training safe in patients with anorexia nervosa? A meta-analysis. Physiotherapy. 2013;99(1):1-11.

83. Schuch FB, Deslandes AC, Stubbs B, Gosmann NP, Silva CT, Fleck MP. Neurobiological effects of exercise on major depressive disorder: a systematic review. Neurosci Biobehav Rev. 2016;61:1-11.

84. Kallies G, Rapp MA, Fydrich T, et al. Serum brain-derived neurotrophic factor (BDNF) at rest and after acute aerobic exercise in major depressive disorder. Psychoneuroendocrinology. 2018;102:212-5.

85. Kerling A, Kuck M, Tegtbur U, et al. Exercise increases serum brain-derived neurotrophic factor in patients with major depressive disorder. J Affect Disord. 2017;215:152-5.

86. Dinoff A, Herrmann N, Swardfager W, Gallagher D, Lanctot KL. The effect of exercise on resting concentrations of peripheral brain-derived neurotrophic factor (BDNF) in major depressive disorder: a meta-analysis. J Psychiatr Res. 2018;105:123-31.

87. Pajonk FG, Wobrock T, Gruber O, et al. Hippocampal plasticity in response to exercise in schizophrenia. Arch Gen Psychiatry. 2010;67(2):133-43.

88. Gross J, Vancampfort D, Stubbs B, Gorczynski P, Soundy A. A narrative synthesis investigating the use and value of social support to promote physical activity among individuals with schizophrenia. Disabil Rehabil. 2016;38(2):123-50.

89. Liu M, Wu L, Ming Q. How does physical activity intervention improve self-esteem and self-concept in children and adolescents? Evidence from a meta-analysis. PLoS One. 2015;10(8):e0134804.

90. Bassett-Gunter R, McEwan D, Kamarhie A. Physical activity and body image among men and boys: a meta-analysis. Body Image. 2017;22:114-28.

91. Herring M. Exercise for the management of anxiety and stressrelated disorders. In: Stubbs B, Rosenbaum S, editors. Exercise based interventions for mental illness: physical activity as part of clinical treatment. London: Elsevier; 2018.

92. Naci H, Ioannidis JPA. Comparative effectiveness of exercise and drug interventions on mortality outcomes: metaepidemiological study. BMJ (Clin Res Ed). 2013;347:f5577.

93. Naci H, Salcher-Konrad M, Dias S, et al. How does exercise treatment compare with antihypertensive medications? A network meta-analysis of 391 randomised controlled trials assessing exercise and medication effects on systolic blood pressure. Br J Sports Med. 2018;53(14):859-69.

94. Vancampfort D, Firth J, Schuch FB, et al. Sedentary behavior and physical activity levels in people with schizophrenia, bipolar disorder and major depressive disorder: a global systematic review and meta-analysis. World Psychiatry. 2017;16(3):308-15.

95. Yates T, Khunti K, Wilmot EG, et al. Self-Reported sitting time and markers of inflammation, insulin resistance, and adiposity. Am J Prev Med. 2012;42(1):1-7.

96. Yates T, Davies MJ, Gray LJ, et al. Levels of physical activity and relationship with markers of diabetes and cardiovascular disease risk in 5474 white European and South Asian adults screened for type 2 diabetes. Prev Med. 2010;51(3/4):290-4.
97. Firth J, Siddiqi N, Koyanagi A, et al. The Lancet Psychiatry Commission on improving physical health in people with mental illness. Lancet Psychiatry. 2019;6(8):675-712.

98. Bora E, Akdede BB, Alptekin K. The relationship between cognitive impairment in schizophrenia and metabolic syndrome: a systematic review and meta-analysis. Psychol Med. 2017;47(6):1030-40.

99. Hryhorczuk C, Sharma S, Fulton SE. Metabolic disturbances connecting obesity and depression. Front Neurosci. 2013;7:177.

100. Volkert J, Kopf J, Kazmaier J, et al. Evidence for cognitive subgroups in bipolar disorder and the influence of subclinical depression and sleep disturbances. Eur Neuropsychopharmacol. 2015;25(2):192-202.

101. Kittel R, Brauhardt A, Hilbert A. Cognitive and emotional functioning in binge-eating disorder: a systematic review. Int J Eat Disord. 2015;48(6):535-54

102. Ekkekakis P. Honey, I shrunk the pooled SMD! Guide to critical appraisal of systematic reviews and meta-analyses using the Cochrane review on exercise for depression as example. Ment Health Phys Act. 2015;8:21-36.

103. Morres ID, Hatzigeorgiadis A, Stathi A, et al. Aerobic exercise for adult patients with major depressive disorder in mental health services: a systematic review and meta-analysis. Depress Anxiety. 2019;36(1):39-53.

104. Mitchell AJ, Yadegarfar M, Gill J, Stubbs B. Case finding and screening clinical utility of the Patient Health Questionnaire (PHQ-9 and PHQ-2) for depression in primary care: a diagnostic meta-analysis of 40 studies. BJPsych Open. 2016;2(2):127-38.

105. Hallgren M, Helgadottir B, Herring MP, et al. Exercise and internet-based cognitive-behavioural therapy for depression: multicentre randomised controlled trial with 12-month followup. Br J Psychiatry. 2016;209(5):414-20.

106. Gordon BR, McDowell CP, Hallgren M, Meyer JD, Lyons M, Herring MP. Association of efficacy of resistance exercise training with depressive symptoms: meta-analysis and meta-regression analysis of randomized clinical trials. JAMA Psychiatry. 2018;75(6):566-76.

107. Stubbs B, Chen LJ, Chang CY, Sun WJ, Ku PW. Accelerometerassessed light physical activity is protective of future cognitive ability: a longitudinal study among community dwelling older adults. Exp Gerontol. 2017;91:104-9.

108. Firth JA, Stubbs B, et al. Association between muscular strength and cognition in people with major depression or bipolar disorder and healthy controls. JAMA Psychiatry. 2018;75(7):740-6.

109. Firth J, Stubbs B, Vancampfort D, et al. Grip strength is associated with cognitive performance in schizophrenia and the general population: a UK Biobank study of 476,559 participants. Schizophrenia Bull. 2018;44(4):728-36.

110. Li Z, Peng X, Xiang W, Han J, Li K. The effect of resistance training on cognitive function in the older adults: a systematic review of randomized clinical trials. Aging Clin Exp Res. 2018;30(11):1259-73.

111. Northey JM, Cherbuin N, Pumpa KL, Smee DJ, Rattray B. Exercise interventions for cognitive function in adults older than 50: a systematic review with meta-analysis. Br J Sports Med. 2018;52(3):154-60.

112. Gregory A, Mallikarjun P, Upthegrove R. Treatment of depression in schizophrenia: systematic review and meta-analysis. Br J Psychiatry. 2017;211(4):198-204.

113. Vancampfort D, Rosenbaum S, Schuch FB, Ward PB, Probst M, Stubbs B. Prevalence and predictors of treatment dropout from physical activity interventions in schizophrenia: a meta-analysis. Gen Hosp Psychiatry. 2016;39:15-23.

114. Gordon BR, McDowell CP, Lyons M, Herring MP. The effects of resistance exercise training on anxiety: a meta-analysis and 
meta-regression analysis of randomized controlled trials. Sports Med. 2017;47(12):2521-32.

115. Lees C, Hopkins J. Effect of aerobic exercise on cognition, academic achievement, and psychosocial function in children: a systematic review of randomized control trials. Prev Chronic Dis. 2013;10:E174.

116. Wilens TE, Adler LA, Adams J, et al. Misuse and diversion of stimulants prescribed for ADHD: a systematic review of the literature. J Am Acad Child Adolesc Psychiatry. 2008;47(1):21-31.

117. Slade SC, Dionne CE, Underwood M, Buchbinder R. Consensus on exercise reporting template (CERT): explanation and elaboration statement. Br J Sports Med. 2016;50(23):1428-37.

118. Cipriani A, Zhou X, Del Giovane C, et al. Comparative efficacy and tolerability of antidepressants for major depressive disorder in children and adolescents: a network meta-analysis. Lancet. 2016;388(10047):881-90.

119. Czosnek L, Lederman O, Cormie P, Zopf E, Stubbs B, Rosenbaum S. Health benefits, safety and cost of physical activity interventions for mental health conditions: a meta-review to inform translation efforts. Ment Health Phys Act. 2018. https:// doi.org/10.1016/j.mhpa.2018.11.001.

120. Stubbs B, Koyanagi A, Hallgren M, et al. Physical activity and anxiety: a perspective from the World Health Survey. J Affect Disord. 2017;208:545-52.

121. Vancampfort D, De Hert M, Stubbs B, et al. A systematic review of physical activity correlates in alcohol use disorders. Arch Psychiatr Nurs. 2015;29(4):196-201.

122. Vancampfort D, Vanderlinden J, Stubbs B, et al. Physical activity correlates in persons with binge eating disorder: a systematic review. Eur Eat Disord Rev. 2014;22(1):1-8.

123. Gummer R, Giel KE, Schag K, et al. High levels of physical activity in anorexia nervosa: a systematic review. Eur Eat Disord Rev. 2015;23(5):333-44.

124. Choi KW, Chen CY, Stein MB, et al. Assessment of bidirectional relationships between physical activity and depression among adults: a 2-sample Mendelian randomization study. JAMA Psychiatry. 2019;74(4):399-408.

\section{Affiliations}

\section{Garcia Ashdown-Franks ${ }^{1,2,3}$ - Joseph Firth ${ }^{4,5,6} \cdot$ Rebekah Carney $^{7} \cdot$ Andre F. Carvalho $^{8} \cdot$ Mats Hallgren $^{9}$. Ai Koyanagi ${ }^{10}$. Simon Rosenbaum ${ }^{11,12}$. Felipe B. Schuch ${ }^{13}$. Lee Smith ${ }^{14}$. Marco Solmi ${ }^{15}$. Davy Vancampfort ${ }^{16,17}$. Brendon Stubbs ${ }^{1,2,3}$}

1 South London and Maudsley NHS Foundation Trust, Denmark Hill, London SE5 8AZ, UK

2 Psychological Medicine, Institute of Psychiatry, Psychology and Neuroscience (IoPPN), King's College London, London, UK

3 Faculty of Kinesiology and Physical Education, University of Toronto, Toronto, ON, Canada

4 NICM Health Research Institute, Western Sydney University, Westmead, Australia

5 Division of Psychology and Mental Health, University of Manchester, Manchester, UK

6 Centre for Youth Mental Health, University of Melbourne, Melbourne, Australia

7 Youth Mental Health Research Unit, Greater Manchester Mental Health NHS Foundation Trust, Manchester, UK

8 Department of Psychiatry, University of Toronto and Centre for Addiction and Mental Health (CAMH), Toronto, ON, Canada
9 Unit of Epidemiology of Psychiatric Conditions, Substance Use and Social Environment (EPiCS), Department of Public Health Sciences, Karolinska Institutet, Stockholm, Sweden

10 Research and Development Unit, Parc Sanitari Sant Joan de Déu, Universitat de Barcelona, Fundació Sant Joan de Déu, Dr Antoni Pujadas, 42, Sant Boi de Llobregat, 08830 Barcelona, Spain

11 School of Psychiatry UNSW, Sydney, Australia

12 Black Dog Institute, Sydney, Australia

13 Department of Methods and Sports Techniques, Universidade Federal de Santa Maria, Santa Maria, Brazil

14 The Cambridge Centre for Sport and Exercise Sciences, Anglia Ruskin University, Cambridge, UK

15 Neurosciences Department, University of Padova, Padua, Italy

16 Department of Rehabilitation Sciences, KU Leuven, Louvain, Belgium

17 UPC KU Leuven, Leuven-Kortenberg, Belgium 